\title{
APLICACIÓN DEL SISTEMA DE PROMOCIÓN DE LA AUTONOMÍA PERSONAL Y ATENCIÓN A PERSONAS EN SITUACIÓN DE DEPENDENCIA EN LA COMUNITAT VALENCIANA: EFECTOS SOBRE EL EJERCICIO DEL DERECHO SOCIAL
}

\author{
Promotional implementation of the personal autonomy and care for \\ dependent situations in the Valencian Community: effects on the exercise \\ of the social right
}

CARMEN MONTALBÁ OCAÑA ${ }^{1}$

\section{Resumen}

Este artículo presenta el estudio de caso realizado sobre el ciclo de vida de la Ley de Promoción de la autonomía personal y atención a situaciones de dependencia (LAPAD) en la Comunitat Valenciana durante el periodo 2006-2012.

El objetivo de esta investigación ha sido identificar las razones que han contribuido a incrementar la brecha entre la formulación legal y su efectiva implementación. La hipótesis de partida, sometida a contraste mediante método etnográfico, propone que la citada ley no puede representar un avance en la afirmación de derechos sociales en el contexto de una débil cultura de la legalidad, como representa el caso valenciano.

La metodología cualitativa y el método etnográfico han guiado el procedimiento de investigación, empleando múltiples técnicas que transitan desde las «narrativas individuales» de informantes clave a los «discursos colectivos» representativos de los diferentes actores considerados (sindicatos, patronal, movimientos sociales, etc.).

Los resultados obtenidos han fijado la atención sobre aquellas dimensiones que remiten al modelo institucional que acoge el derecho social y aquellas condiciones que posibilitan o dificultan su pleno ejercicio, confirmando la hipótesis planteada a partir de la identificación de los siguientes obstáculos en el reconocimiento y ejercicio del derecho: la relación público-privado en la política social valenciana, la relación de la administración y la ciudadanía valenciana (rendición de cuentas y transparencia), el conflicto competencial y los mecanismos de defensa del derecho que conducen a la judicialización de la política.

Palabras clave: políticas públicas, derechos sociales, situación de dependencia y cultura de la legalidad.

\begin{abstract}
This paper presents a case study made along the life cycle of the Law for Promotion of Personal Autonomy and Care for Dependent Situations (LAPAD) in the Valencian Community during the period 2006-2012.

The objective of this research was to identify the reasons that have contributed to increase the gap between the legal formulation and its effective implementation. The initial hypothesis, subjected to contrast by the ethnographic method, proposes that the Act can not represent a breakthrough in the assertion of rights in the context of a weak culture of legality, as it is the case of Valencia.

The qualitative methodology and the ethnographic methodology have guided this research process, using multiple techniques: from the «single narrative» of key informants to the «collective discourses» represented by different actors (trade unions, employers, social movements, etc.).

The results have pointed out those dimensions that refer to the institutional model that welcomes social law and the conditions that enable or impede its full exercise. In the recognition and exercise of the law, these results have confirmed the initial hypothesis from the following clear identification of obstacles: the public-private relationship in Valencian social policy, the ratio among the public administration and Valencian citizenship (accountability and transparency), and the conflict and jurisdictional law defense mechanisms that lead to criminalization of politics.
\end{abstract}

Keywords: public policy, social rights, dependency and culture of legality.

Recibido: 02/09/2013

Aceptado: 11/11/2013

Publicado: 03/12/2013

1. Dpto. Trabajo Social y Servicios Sociales. Facultad de Ciencias Sociales. Universitat de València. Despacho D.09. Primera planta. Tfno. 9638288 07. Carmen.Montalba@uv.es. 


\section{Introducción}

El presente artículo sintetiza un trabajo de más de cinco años de estudio sobre el ciclo de vida de la Ley de promoción de autonomía personal y atención a personas en situación de dependencia (LAPAD) en la Comunitat Valenciana.

El objeto de estudio ha evolucionado en estos años pudiendo distinguir dos fases en el proceso de investigación: una exploratoria, centrada en el interés por conocer el proceso de gestación de la ley y la negociación de alternativas y una segunda fase, de estudio de caso, coincidente con la etapa de implementación y puesta en marcha de la misma en el territorio valenciano.

La investigación se ha ido enfocando sobre las variables que han intercedido como obstáculos en el reconocimiento y ejercicio del derecho social en el marco de la relación administración-ciudadanía.

Para la historia de la Política Social desarrollada en democracia, esta ley se presenta como un gran avance, con la aspiración de convertir al sistema de servicios sociales en el cuarto pilar de bienestar social. De esta manera, tal y como en su propia exposición de motivos se expresa «configurar un nuevo desarrollo de los Servicios Sociales del país que amplie y complemente la acción protectora de este sistema, potenciando el avance del modelo de Estado social que consagra la Constitución Española, potenciando el compromiso de todos los poderes públicos en promover y dotar los recursos necesarios para hacer efectivo un sistema de Servicios Sociales de calidad, garantistas y plenamente universales».

La LAPAD encarnaba, por tanto, la posibilidad de reforzar al Estado Social con el reconocimiento de un nuevo derecho social y, sobre todo, la posibilidad de reforzar el papel de los poderes públicos como garantes del mismo. Pero, paradójicamente, se ha ido fraguando en un contexto histórico adverso en el que han coincidido la crisis del Estado de Bienestar junto a cambios sociodemográficos (envejecimiento y cambios en los modelos de familia), crisis económica, flexibilización del mercado de trabajo, pérdida de poder de negociación y representación de los agentes sociales, transferencia creciente de algunas competencias a las comunidades autónomas a la par que la recentralización de otras, etc. En este contexto, y tal y como lo expresa Roldán (2001: 2): «(...) se está produciendo una restructuración institucional del mismo y una reorientación ideológica como la privatización de los servicios sociales, un aumento significativo de las organizaciones altruistas, la ruptura del ideal universalista de protección social y el auge de la exclusión social (o «desafiliación», dificultades de acceso a los mecanismos básicos de integración social)».

En definitiva, esta coyuntura ambivalente ha sido capaz de acoger e impulsar una ley con pretensiones para configurar un nuevo sistema de protección 
basado en principios de igualdad, universalidad y el carácter público de las prestaciones y servicios aunque los coletazos de los últimos años han dado claro testimonio que tales principios se reducen a un mero espejismo.

La fase de implementación pone en evidencia, en mayor medida, la paradoja o falta de coherencia entre el nivel normativo o formal y los hechos, con una especial atención a la relación que establecen los diferentes agentes presentes en este escenario. Esto invita a verificar la siguiente hipótesis: la LAPAD no puede representar un avance en la afirmación de los derechos sociales en el marco de una débil cultura de la legalidad ${ }^{3}$, como representa el caso valenciano.

En este sentido, la LAPAD se concreta en un sistema (sistema para la promoción de la autonomía personal y atención a personas en situación de dependencia - SAAD) que cuenta con la colaboración y participación de todas las Administraciones Públicas (Administración General del Estado - AGE, administración autonómica y administración local) aunque también reconoce la participación de la iniciativa privada ${ }^{4}$ y del tercer sector.

Pese a que el objeto de estudio reconoce la relación entre todos estos agentes, hay uno destacado dado el ordenamiento actual de los servicios sociales: la administración autonómica. Por tanto, la propia naturaleza del presente objeto impulsa al estudio de caso centrando la atención en la realidad valenciana y la elección de la etnografía como método y metodologías capaces de aprehender todo el proceso de gestación e implantación de una política en un territorio.

\section{Método y metodología}

El objetivo central de la presente investigación ha sido identificar las razones que nutren la brecha existente entre el texto legal y su efectiva implementación a partir del contraste de la hipótesis ya enunciada, siendo un escenario privilegiado para emprender este análisis la relación administración-ciudadanía.

En la consecución de dicho objetivo, bajo un diseño de investigación abierto, se ha evolucionado desde la citada fase exploratoria (de junio a noviembre de 2006), de carácter descriptiva, en la que se aborda las fases de la política pública relativas a la formulación del problema (problematización de

3. El concepto de «cultura de la legalidad» empleado remite a un sistema coherente entre el plano formal, vinculado al derecho y a las leyes, y el plano real de la implementación de las políticas públicas, concepto incluido en el de calidad democrática y buen gobierno, que a su vez acoge a otros términos como rendición de cuentas y transparencia.

4. El artículo 6.2 dice el «Sistema se configura como una red de utilización pública que integra, de forma coordinada, centros y servicios, públicos y privados». 
la «situación de dependencia») y la negociación de alternativas (gestación de la LAPAD), a una fase de estudio de caso (de diciembre de 2006 a diciembre de 2010), con un mayor carácter interpretativo, en la que se analiza la implementación de la ley y se contrasta la hipótesis.

Las dimensiones de descripción y análisis en la fase exploratoria han gravitado sobre:

a. La problematización de la situación de dependencia: qué es y cómo se reconoce.

b. Negociación de alternativas, cómo se despliegan las respuestas al problema: marco normativo, procedimiento de reconocimiento del derecho (agentes que intervienen), e implantación del catálogo de servicios y prestaciones.

En la fase de estudio de caso se profundiza en lo enunciado y se intenta dar cuenta mayormente de la implementación de la ley a través de:

- El análisis longitudinal y comparativo (entre diferentes territorios) a partir de los datos que proporciona el Sistema de Información del SAAD mensualmente que evidencian el grado de implantación y, por tanto, la cobertura y atención al derecho. Entre otros, los indicadores estadísticos analizados han sido: la evolución de las solicitudes, las solicitudes efectivamente valoradas frente a las prestaciones reconocidas, el régimen de compatibilidades mediante la ratio prestaciones por persona beneficiaria, el peso de las prestaciones económicas frente a los servicios, el peso de la prestación de cuidados en el entorno familiar, etc.

- Elementos de carácter institucional que inciden en el reconocimiento y ejercicio del derecho (qué actores, en base a qué reglas del juego, qué valores y qué cultura alimentan), siendo dimensiones de análisis básicas: los niveles de protección y financiación; sistema de información; estructura de coordinación interadministrativa; gobernabilidad; acreditación de centros y servicios; intensidad y compatibilidad de los mismos; así como el procedimiento desplegado en el reconocimiento del derecho en la Comunitat Valenciana.

- El grado de formalidad jurídica de las actividades administrativas y, por tanto, las garantías de protección jurídica de la ciudadanía frente a sus actos a partir de la busqueda de indicadores de rendición de cuentas legal y administrativa, tales como: la transparencia, la responsabilidad pública, cumplimiento del procedimiento administrativo, 
etc. así como las estrategias desplegadas en la defensa del derecho: rendición de cuentas societal y horizontal.

Para poder abordar todas estas aristas que dan forma al objeto de estudio, la estrategia metodológica ha evolucionado en paralelo: desde la fase exploratoria con mayor peso de las metodologías cualitativas guiadas por paradigmas comprensivos e interpretativos con el protagonismo de entrevistas semiestructuradas (individuales y grupales) (ver tabla 1 y 2) a la fase de estudio de caso con la aplicación del método etnográfico, orientado por paradigmas críticos, en la que se amplía y diversifica la utilización de técnicas, entre otras: el análisis del discurso de diferentes registros etnográficos, observación y análisis del discurso en comunidades virtuales, auto-observación, observación

Tabla 1: Técnicas de la Fase Exploratoria, 14 entrevistas semiestructuradas

\begin{tabular}{|c|c|}
\hline $\begin{array}{l}\text { INFORMANTES } \\
\text { CLAVE ÁMBITO } \\
\text { PÚBLICO }\end{array}$ & ENTIDAD \\
\hline 3 & $\begin{array}{l}\text { Direcciones territoriales: } \\
\text { - Jefatura de Acción Social de Alicante } \\
\text { - Jefatura de Mayores de Valencia } \\
\text { - Jefatura de Discapacitados/as de Castellón }\end{array}$ \\
\hline 1 & IVADIS \\
\hline 2 & Residencia Pública Mayores y discapacitados/as \\
\hline 1 & Administración local con SAD propio \\
\hline 1 & Centro de Día, CRIS. \\
\hline $\begin{array}{l}\text { INFORMANTES } \\
\text { CLAVE ÁMBITO } \\
\text { PRIVADO } \\
\end{array}$ & ENTIDAD \\
\hline 2 & $\begin{array}{l}\text { - Empresa representativa ámbito privado residencial que opera a } \\
\text { nivel autonómico con mayores. } \\
\text { - Entidad sin ánimo de lucro representativa del ámbito } \\
\text { residencial que opera a nivel autonómico con personas } \\
\text { discapacitadas. }\end{array}$ \\
\hline 2 & $\begin{array}{l}\text { - Empresa representativa del ámbito privado, SAD que opera a } \\
\text { nivel provincial (Valencia, Castellón y Alicante). } \\
\text { - Entidad sin ánimo de lucro del SAD que opera a nivel } \\
\text { provincial (Valencia, Castellón y Alicante). }\end{array}$ \\
\hline 1 & $\begin{array}{l}\text { Empresa y/o entidad sin ánimo de lucro representativa a nivel } \\
\text { comarcal o local-centro de día o CRIS. }\end{array}$ \\
\hline 1 & $\begin{array}{l}\text { Empresa y/o entidad sin ánimo de lucro representativa a nivel } \\
\text { comarcal o local - Teleasistencia. }\end{array}$ \\
\hline
\end{tabular}

Fuente: Elaboración propia 
Tabla 2: Técnicas de la Fase Exploratoria, 2 entrevistas grupales

\begin{tabular}{|c|c|}
\hline $\begin{array}{l}\text { GRUPO FOCALIZADO A } \\
\text { PROFESIONALES } \\
\text { CRITERIOS DE SELECCIÓN: } \\
\text { Conocimientos profesionales } \\
\text { sobre el presente de la atención } \\
\text { a situación de dependencia y } \\
\text { su análisis o reflexión sobre la } \\
\text { futura ley. }\end{array}$ & $\begin{array}{l}\text { - Colegio profesional de Trabajadores Sociales (2 } \\
\text { representantes) } \\
\text { - Asociación de directores y gerentes de SS.SS. (1 } \\
\text { representante) } \\
\text { - Sindicato/s mayoritarios - UGT y CCOO } \\
\text { (Federación de Sanidad, Enseñanza y } \\
\text { Administración Pública) (3 representantes) }\end{array}$ \\
\hline $\begin{array}{l}\text { GRUPO FOCALIZADO A } \\
\text { EMPRESAS } \\
\text { CRITERIOS DE SELECCIÓN: Su } \\
\text { presencia en el mercado vinculado } \\
\text { a la atención de personas en } \\
\text { situación de dependencia, y su rol } \\
\text { de agentes social protagonista en } \\
\text { espacios de negociación. }\end{array}$ & $\begin{array}{l}\text { - Patronales de cooperativas, FEVECTA. } \\
\text { - Asociación de Empresarios de Economía Social de } \\
\text { SS.SS. de la C.V. AEES } \\
\text { - Gesmed } \\
\text { - Secopsa } \\
\text { - Asociación Empresarial de Residencias y Servicios } \\
\text { Sociales de la Comunitat Valenciana (AERTE) } \\
\text { - Asociación de Residencias no Lucrativas C.V. } \\
\text { - } \text { (Lares Comunitat Valenciana) } \\
\text { Patronal de discapacitados psíquicos FEAPS }\end{array}$ \\
\hline
\end{tabular}

Fuente: Elaboración propia

Tabla 3: Técnicas de la Fase estudio de caso, 8 entrevistas semiestructuradas a expertos/as juristas

\begin{tabular}{|c|l|}
\hline $\begin{array}{c}\text { NÚM.INFORMANTES } \\
\text { CLAVE }\end{array}$ & \multicolumn{1}{|c|}{ ENTIDAD } \\
\hline 1 & $\begin{array}{l}\text { Organizaciones legalmente constituidas para la defensa de } \\
\text { los derechos ciudadanos: Sindicatura de Greuges y Colegios } \\
\text { de Abogados (turnos de oficios). }\end{array}$ \\
\hline 4 & $\begin{array}{l}\text { Abogados de organizaciones sindicales y ONGs que están } \\
\text { prestando servicios en este ámbito. }\end{array}$ \\
\hline 2 & Expertos/as juristas del ámbito académico. \\
\hline 1 & Magistrados del Tribunal Superior Justicia. \\
\hline
\end{tabular}

Fuente: Elaboración propia

participante, entrevistas informales, entrevistas semiestructuradas (orientadas a agentes sociales y a profesionales), entrevistas a expertos/as juristas que asesoran en la defensa del derecho y talleres grupales aplicando la técnica del tetralema (ver tabla 3 y 4 ). 
Tabla 4: Técnicas de la Fase estudio de caso, 2 talleres grupales aplicando la técnica del Tetralema

\begin{tabular}{|c|l|}
\hline Objetivo & $\begin{array}{l}\text { Profundizar en los significados que se atribuyen a aquello que se } \\
\text { calificaba de «público» y de «privado»: qué prácticas y sentidos } \\
\text { aglutinan estos términos y cómo interfieren dichos significados en el } \\
\text { ejercicio del derecho }\end{array}$ \\
\hline $\begin{array}{l}\text { Taller de } \\
\text { Valencia }\end{array}$ & $\begin{array}{l}\text { Plataforma Valenciana en defensa de la LAPAD, constituido por un total de } \\
\text { ocho personas, fundamentalmente profesionales del sector y algún familiar } \\
\text { de persona afectada. }\end{array}$ \\
\hline Taller de & $\begin{array}{l}\text { Plataforma Alicante en defensa de la LAPAD, con una participación } \\
\text { de cuatro personas pertenecientes a colectivos de enfermos crónicos y } \\
\text { personas discapacitadas. }\end{array}$ \\
\hline
\end{tabular}

Fuente: Elaboración propia

\section{Resultados}

En este epígrafe se presentan los resultados destacados de cada una de las fases del ciclo de vida de la ley analizadas:

\subsection{Resultados de la fase de formulación y de alternativas: problematización de la dependencia y respuesta institucional}

En esta fase se ha analizado cómo se construye como problema la «situación de dependencia», su legitimación y la respuesta desde las políticas públicas. Así, en términos discursivos, dado que la ley remite a una "situación» (enfoque situacional) y no a un colectivo, se puede afirmar que se ha manejado una definición próxima al denominado modelo social, no prestando atención a los rasgos que sirven para identificar a la persona con «discapacidad o diversidad funcional» sino a las circunstancias en las que la persona se encuentra y que limitan sus posibilidades de autonomía, en definitiva, las razones de su construcción social.

Sin embargo, la investigación realizada demuestra que la operativización del concepto a través de los instrumentos de valoración de la dependencia ha seguido enmarcándola en el denominado modelo médico-rehabilitador. Como afirman Pié (2012) y Romañach (2012), la ley genera una categoría que filtra a la población entre quienes pueden o no pueden acceder al sistema de distribución basado en las necesidades. De esta manera, el concepto empleado estaría, en la práctica, huyendo de una visión antropológica y ontológica de las situaciones de dependencia como condiciones consustanciales al ser humano, con su expresión más evidente en la infancia y en la vejez. 
En la construcción social del problema y su legitimación también ha interesado identificar qué tipos de respuestas se articulan. En este sentido, la aprobación de la LAPAD ha supuesto el paso fundamental para ofrecer una intervención desde la esfera pública. Tradicionalmente, la actividad del cuidado y la atención a situaciones de dependencia quedaban relegadas al ámbito privado, fruto también de una división sexual del trabajo en la cual se hacía a la mujer responsable de la prestación del socorro y cuidado de sus seres queridos y familiares. La intervención pública en España se ha sostenido en una asistencia social vinculada a situaciones de emergencia y carencia, prestada con cierto carácter arbitrario y siempre sujeta a la prueba de suficiencia de recursos.

La LAPAD reconoce como derecho subjetivo la atención a las situaciones de dependencia y, de esta forma, les otorga un nuevo valor o carácter a través de su consideración como derecho, desplazando la obligación al ámbito público, gubernamental.

Finalmente, un efecto no previsto en la formulación de la política pública pero real en sus consecuencias ha sido la conversión de la situación de dependencia como paradigma de la «autonomía». La ideología liberal siempre ha sido temerosa del intervencionismo estatal, entre otros argumentos, porque es generador de dependencias, de ciudadanías pasivas, en condición permanente de súplica y demanda a la administración para que ésta le resuelva los problemas. Sin embargo, la LAPAD ha conseguido el efecto contrario en las personas que padecen una situación de dependencia, gracias al reconocimiento del derecho y a la asunción de éste por parte de la ciudadanía.

Un ejemplo de cómo la deficiente aplicación de esta ley ha conseguido recuperar la identidad republicana de la ciudadanía activa ${ }^{5}$ es la conformación de múltiples plataformas que reivindican el derecho social a la atención y la responsabilidad pública. El movimiento social rompe con la idea liberal de

5. «(...) Los ciudadanos pasivos son aquéllos que se limitan a cumplir con un mínimo de obligaciones, aunque en momentos efímeros de emoción colectiva puedan manifestarse públicamente. Para ellos el ejercicio de la virtud cívica consiste en la obediencia rutinaria a la autoridad legítima, es decir, el pago de contribuciones sin evasión fiscal detectable, el relativo buen comportamiento en la vía pública, y demás expresiones de buena conducta cívica aceptable, amén de su presencia en las urnas. Los ciudadanos activos, por su parte, son aquéllos que, sin ser profesionales de la política, intervienen en la esfera pública para mejorar las condiciones de la vida democrática, ejercer su propia libertad y, sobre todo, cultivar la virtud suprema de la república, la fraternidad. Los ciudadanos activos son, esencialmente, proactivos, es decir, toman iniciativas para cumplir estos fines, al margen o más allá de situaciones que les hayan perjudicado o dañado» (Giner, 2005: 19). 
una ciudadanía pasiva, hace frente a la tesis de la infantilización ${ }^{6}$ donde lo que se pretende es amamantarse del Estado, la «dependencia de la ayuda social»y, por el contrario, se comporta como el adulto que se ha atrevido a saber (aude sapere), que ha tomado conciencia crítica de lo que esta ley presupone y ha activado dispositivos de reivindicación para poder ejercer el nuevo derecho que se les reconoce así como mecanismos pedagógicos para su divulgación.

En definitiva, una situación personal de dependencia en las actividades de la vida cotidiana no se traduce en una situación de dependencia/pasividad frente a la administración en el sentido liberal sino más bien de la ciudadanía activa como sujeto político, que reivindica su posición en el espacio público y da un paso importante hacia la gobernabilidad del Estado de Bienestar: «Nunca esta tradición pudo hacer ver de qué manera era posible trasladar al ámbito público las lecciones que en privado se aprenden sobre el carácter y enriquecer así la política con peso psicológico real» (Sennett, 2003: 132).

Este aspecto, identificado en la fase de legitimación del problema, constituirá un factor importante en la conformación de una fuerte rendición de cuentas societal.

Por su parte, en la fase de alternativas se ha analizado el proceso de negociación y debate acontecido en la gestación de la ley y el desarrollo de una política de atención a situaciones de dependencia. El centro de la discusión se situó en su encuadramiento institucional, competencial y de financiación para asegurar el derecho. Las discusiones académicas, políticas y tecnocráticas pivotaban alrededor de: financiación mediante Seguridad Social o por vía impositiva, demarcación sectorial en el sistema de servicios sociales o en el sistema sanitario, configuración como Ley Básica o su asunción autonómica, y predominio de un sistema público frente a un sistema mixto.

El proceso de negociación se inició con la recomendación que el Defensor del Pueblo emitió en el año 2000 aludiendo a la «oportunidad de implantar en España un sistema de protección a la dependencia en el marco de la Seguridad Social» (Rodríguez; Arriba; Marban y Salido, 2005). A partir de este momento surgen toda una serie de declaraciones y acuerdos, fruto de la negociación política en la que se enmarca el debate: la Comisión Parlamentaria de seguimiento del Pacto de Toledo (mayo de 2000), el Acuerdo para la Mejora y Desarrollo del Sistema de Protección Social (3 de abril de 2001), la renovación del Pacto de Toledo (2003), el Acuerdo Competitividad, Empleo y Cohesión Social (julio de 2004), la publicación del Libro Blanco de la Dependencia (2005) hasta

6. «Los pensadores liberales han supuesto que la dependencia, en particular la dependencia de los poderes públicos, hace que los adultos se comporten como niños» (Sennett, 2003: 111). 
llegar, finalmente, al Acuerdo de la situación protectora: Sistema Nacional de Dependencia (diciembre de 2005). Será en este último donde se plantea el modelo que acogerá al sistema mediante una ley que tenga la condición de norma básica, que promueva la autonomía personal y garantice el derecho a ser atendido en situaciones de dependencia, y en la que se establecerán las condiciones básicas y los requisitos de acceso a los servicios y prestaciones en condiciones de equidad en todo el territorio del Estado. El Sistema Nacional de la Dependencia ${ }^{7}$ se configuraría como una nueva modalidad de protección social, que debería regir bajo los siguientes principios: carácter universal y público de las prestaciones; acceso a las prestaciones de dependencia en condiciones de igualdad y no discriminación; equidad en la distribución territorial de los recursos; carácter universal y público de las prestaciones; acceso a las prestaciones de dependencia en condiciones de igualdad y no discriminación y la equidad en la distribución territorial de los recursos. Estos principios sirvieron para alimentar la expectativa de lo que algunos/as llamarían cuarto pilar del Estado de Bienestar.

En los pasos posteriores que correspondieron al trámite parlamentario, de discusión y participación, que discurrieron desde el Consejo de Ministros (23 de diciembre de 2005) hasta la publicación en el Boletín Oficial del Estado en diciembre de 2006, la cuestión competencial apareció como un tema central en el debate político, produciéndose diferentes enmiendas ${ }^{8}$ a la totalidad del proyecto de ley que gravitaban sobre la injerencia del Estado al establecer una norma básica en un ámbito competencial autonómico.

La legitimidad competencial se arrastrará hasta nuestros días como un obstáculo evidente en la implementación de esta ley.

Finalmente, con el acuerdo de un sistema de protección universal e igualitario, con la aspiración por parte de la AGE de regular las condiciones básicas y el contenido mínimo que además debería someterse al marco de cooperación interadministrativa con la constitución del Consejo Territorial, se gestó una ley que ha representado el paso de un modelo de protección asistencial a un modelo de carácter universalista o continental ya que compartía con éstos, al menos sobre el papel: el carácter universal, el sistema de copago, la

7. Denominación que tras el proceso de alegaciones pasó a ser Sistema de Promoción de la Autonomía y Atención a las situaciones de dependencia (SAAD).

8. Se presentaron las enmiendas a la totalidad del Grupo Parlamentario Vasco (PNV-EAJ), CIU y Eusko Alkartasuna (Grupo Mixto) siendo la invasión de competencias autonómicas exclusivas en servicios sociales la razón aducida para votar en contra de la LAPAD cuando ésta se aprobó por el Pleno del Congreso de los Diputados el 30 de noviembre de 2006 (Diario de Sesiones del Congreso de los Diputados n 219, 30 Noviembre de 2006). 
responsabilidad pública, la preferencia de servicios y la orientación a dignificar la figura de la persona cuidadora.

\subsection{Resultados de la fase de implementación}

Resulta complejo sintetizar los resultados principales de esta fase dado que se ha analizado un amplio espectro de factores que tienen incidencia directa sobre el reconocimiento y ejercicio del derecho social, siendo éstos: la financiación, el sistema de información, la estructura institucional de coordinación y gestión, la intensidad y compatibilidad entre servicios y prestaciones, la relación público-privada y, finalmente, el procedimiento relativo al reconocimiento del derecho. A continuación se señalan sólo algunos de los resultados que se considera que tienen una incidencia más notable en el reconocimiento y ejercicio del derecho, pues además convergen en cada una de las dimensiones analizadas.

a) El débil papel de tutela y garante del derecho de la Administración General del Estado

Todos los diagnósticos ${ }^{9}$ realizados en torno a la desigual aplicación territorial de la ley apuntan a una misma dirección: falta de coordinación interadministrativa y la necesidad de un mayor protagonismo de la administración central para asegurar la necesaria homogeneidad. Esta demanda es como pedirle "peras al olmo» porque si la AGE cumple su papel de tutela y liderazgo puede ser acusada de inconstitucionalidad por interferir en el espacio de competencia autonómica mientras que, por otro lado, debe garantizar condiciones de «igualdad» en todo el territorio.

La fórmula innovadora del Consejo Territorial (C.T.), el cual pretendía lograr la co-implicación de todas las administraciones, ha desempeñado un rol paradójico y claramente inoperante. Esta fórmula no ha conducido al éxito porque el interés general no ha coincidido siempre con el interés de todas las administraciones representadas y de todo el espectro partidista que las gobernaba.

9. El informe encargado a la Agencia Estatal de Evaluación de las Políticas Públicas y la Calidad de los Servicios (2009), el I Dictamen de la Asociación Estatal de Directores y Gerentes de SS.SS. (2008), Estudio de la Fundación Alternativas (Kahale, 2009), Informe balance realizado por CC.OO. (2009), Estudio comparativo autonómico sobre los desarrollos normativos promovido por el CERMI (2009), Informe de Gobierno para estos cinco años de aplicación de la LAPAD (VV.AA., 2011: 583). 
De la misma manera, las autonomías interpretaban de formas múltiples su responsabilidad con el impulso de la ley según el partido que las gobernaba, según el peso histórico de los servicios sociales o según la estructura institucional que se deseara desplegar en torno a la misma, entre otros factores. Nos situábamos pues ante un problema de difícil solución y que debe trascender el debate formulado en términos simplistas de «centralismo vs autonomismo» como si tomar posición por uno u otro de los modelos de estado y gobierno resolviera el problema de fondo.

Si el Consejo Territorial hubiera sido capaz de concretar al máximo los acuerdos de los que era responsable y de comprometer a todas las administraciones en la mutua supervisión y vigilancia, hubiéramos estado ante una nueva fórmula madura para superar el centralismo del gobierno central y los particularismos autonómicos en pro de la igualdad. En definitiva, se trataba de experimentar una nueva fórmula que requería de la convicción y esfuerzo de las diferentes administraciones y gobiernos implicados, tal y como lo expresa el Informe de la Comisión de Expertos (Cervera, Herce, López, Rodríguez y Sosvilla, 2009): «El objetivo del Consejo Territorial es robustecer la coordinación en un sistema descentralizado y, como tal, hay que contemplar su desarrollo en el futuro de forma que el SAAD no sea un conjunto de diecinueve subsistemas, sino un único sistema, coherente en su diversidad, que garantice en todo el territorio los principios básicos de la ley: universalidad, igualdad, transparencia, cooperación y eficacia. En este sentido, sería deseable el perfeccionamiento de los mecanismos de un gobierno cooperativo que conjugue diversidad y armonización en aras de la creación de un marco global de acción integrada».

En conclusión, la mejor manera de aumentar el control suponía ampliar las exigencias en rendición de cuentas, acordando mecanismos comunes de auditoría y sanciones ante los incumplimientos. Incluso si el Comité Consultivo estuviera ampliado por organizaciones y plataformas ciudadanas, el control sobre las administraciones también podría ejercerse desde abajo. Para ello resultaba necesario seguir mejorando los mecanismos de información y transparencia respecto a la gestión concreta realizada por las diferentes administraciones, siendo necesaria la mejora y ampliación de los indicadores ofrecidos en las estadísticas mensuales del SISAAD. Entre los más reivindicados por colectivos y profesionales figuran los propios plazos de tramitación y espera así como los mecanismos de sanción frente a las desviaciones como parte de la rendición de cuentas. 
b) La financiación

Uno de los aspectos que ha debilitado la tutela del derecho por parte de la AGE es la propia estructura de financiación que debía acogerle. La LAPAD caracteriza el modelo de financiación como estable, suficiente, sostenido en el tiempo y equitativo pero ninguna de estas condiciones se ha confirmado en su efectiva implementación.

La investigación realizada ha demostrado que lo que ha tenido graves efectos sobre el derecho no es sólo el cuánto sino también el cómo la ingeniería de financiación desplegada ha sido sometida a cambios permanentes.

Con el fin de realizar una aproximación esclarecedora del galimatías que supone la citada ingeniería financiera, ha resultado necesario centrarse en tres cuestiones mutuamente imbricadas: la totalidad del dinero aportado (fondos), las responsabilidades contributivas que debían o podían asumir las diversas administraciones públicas por niveles territoriales (niveles de protección) y, por último, la asignación distributiva que con carácter autonómico se hacía de parte de los fondos estatales, conforme a una serie de criterios, en continua y cambiante evolución.

En la LAPAD se denomina nivel mínimo de protección a los fondos que son garantizados por la AGE. Al margen de cualquier otra aportación financiera que pudiera realizarse, como mínimo, la administración central se comprometía a la fijación de una cantidad económica mensual por cada una de las personas beneficiarias reconocidas según su grado y nivel de dependencia valorado. En la ley se determina que la liquidación de estos fondos se realizaría mensualmente, en función de los datos de reconocimiento efectivo del derecho a las prestaciones que cada comunidad autónoma registrara. Quizás fue ésta la razón que motivó que algunas autonomías realizaran «valoraciones de oficio», viendo de esta manera incrementar sus estadísticas y percibiendo, consecuentemente, la oportuna financiación estatal.

Otra de las perversiones que el sistema consentía es el desfase entre la recepción del dinero por parte de las autonomías y la transmisión del mismo a sus legítimos/as destinatarios/as. Ya se ha mencionado que los pagos de la AGE se realizan mensualmente por cada persona que ha sido valorada como dependiente, aunque esta última aún no haya recibido ninguna prestación o servicio. Por lo tanto, las administraciones autonómicas podrían disponer de crédito sin intereses para poder acometer otros pagos u obras. Es más, estos entes públicos periféricos podían tener la tentación de demorar los trámites para otorgar la prestación o servicio correspondiente pues, de esta manera, conseguían seguir disponiendo libremente de una financiación adicional sin 
coste financiero en momentos donde disponer de liquidez para las arcas públicas ha supuesto un grave problema.

Posiblemente por este motivo se aprobó el R.D. 99/2009 que junto a que el procedimiento debía ser iniciado a solicitud de la persona interesada, proponía cierta aclaración respecto de cómo se habría de justificar la valoración de las personas en situación de dependencia para poder recibir las transferencias monetarias del nivel mínimo, así como que sólo se abonarían las resoluciones de reconocimiento que incluyeran el Programa Individual de Atención (PIA).

Es necesario destacar lo difícil que ha resultado investigar cuáles han sido las transferencias exactas que ha realizado la AGE a cada comunidad autónoma por este concepto, dada la falta de transparencia informativa por parte de ambas instituciones, emisora y receptora.

Por su parte, el nivel de protección acordado, que teóricamente debía ser determinado entre la administración central y las administraciones autonómicas a través de los convenios bilaterales previstos en la ley, plantea dos claras restricciones unilaterales: en primer lugar, la contribución estatal venía predeterminada y, en segundo lugar, se le exigía a cada comunidad autónoma que debía contribuir a la financiación «acordada» con una cantidad al menos igual a la aportada por la AGE incluyendo los fondos del nivel mínimo que fueran otorgados.

Sin embargo, no ha existido equilibrio entre la aportación de las administraciones (central y autonómicas). La explicación residía en diferenciar el denominado como Gasto Consolidado frente al Gasto Nuevo (VV.AA., 2011: 185, 196-199): «En la LAPAD se establece que la aportación de las comunidades autónomas será para cada año, al menos igual a la de la Administración General del Estado en concepto de nivel mínimo y nivel acordado (Art. 32), entendiéndose que esta «nueva financiación» debe ir destinada para el gasto "nuevo", necesario para cumplir los objetivos de la Ley. En consecuencia se trata de fondos nuevos tanto para el Estado como para las comunidades, que deberían añadirlo al gasto que ya venían realizando antes de la Ley». Ese gasto ya realizado, que tenía sus vías de financiación, es el denominado Gasto Consolidado. De hecho, cuando la ley daba sus primeros pasos, las comunidades autónomas presentaban sus tremendos logros que en ciertos casos solamente respondían a hacer aflorar como gasto nuevo lo que realmente era gasto consolidado ${ }^{10}$.

10. Los primeros años de aplicación, según calendario fijado en la ley, se debía reconocer a la población Gran Dependiente, que en su gran mayoría ya estaba siendo objeto de atención por algún recurso del sistema de servicios sociales. 
Los criterios de distribución autonómica del nivel acordado han de ser fijados, mediante acuerdo del Consejo Territorial, a través de los denominados como Marco de Cooperación Interadministrativa (MCI). Es evidente que los MCI se fueron estableciendo para ir acompañando la aplicación de la ley, aportando dinero antes del cumplimiento de objetivos. La antelación en el desembolso se justificaba por el compromiso adquirido por las autonomías. No obstante, la ejecución de la LAPAD ya señalaba algo con claridad: la voluntad de desarrollar la ley no era la misma en todos los territorios del estado español. Parecía atisbarse entonces que el MCI debía pasar de ser una mera comparsa en la ejecución de la ley a una brújula que estableciera cuáles son las razones que justifican la recepción de los fondos y, por tanto, que obligara a hacer cambios de rumbo importantes a determinadas autonomías que parecían claramente «desorientadas«.

Tras varios anuncios sobre una gran reforma de los MCIs, se pospuso ésta al mismo tiempo que se aprobaba un programa de carácter plurianual (2010-2013), publicado en el BOE de 21/07/2010 11 el cual incorporaba los cambios en los criterios como consecuencia, además, de la aprobación de una nueva ley de financiación de las autonomías. Esta reforma modificaba uno de los ítems que más pesaba en el Fondo General y, por tanto, en el conjunto total del nivel acordado: el volumen de población potencialmente dependiente (PPD). El reparto de Fondos entre las CC.AA. correlacionaba positivamente con esta variable: a más población potencialmente dependiente, más financiación (independientemente del esfuerzo que estuviera realizando en el efectivo reconocimiento del derecho). Sin embargo, mediante los cambios del nuevo marco se intentaba ligar más la financiación a su efectivo cumplimiento, incrementando el pago en función de los Planes Individuales de Atención aprobados, como se expresa en las ponderaciones de los diferentes criterios de la Tabla 5.

Estos cambios sí que han logrado que el MCI sea esa brújula que desea marcar el rumbo y el ritmo para lograr una plena implantación de la ley. Aquellas comunidades que no habían sabido o querido enderezar el timón son fácilmente identificables en la siguiente tabla, expresando el poco esfuerzo realizado con el consiguiente sufrimiento de sus ciudadanos/as en situación de dependencia.

11. Resolución de 15 de julio de 2010, del Instituto de Mayores y Servicios Sociales, por la que se publica el Acuerdo de Consejo de Ministros de 9 de julio de 2010, por el que se aprueba el marco de cooperación interadministrativa y criterios de reparto de créditos de la AGE para la financiación durante 2010-2013 del nivel acordado. 
Tabla 5: Criterios de reparto de los fondos destinados al nivel acordado en el MCI de 2010

\begin{tabular}{|c|c|c|c|c|c|c|}
\hline & CRITERIOS & FONDOS 2010 & 2010 & 2011 & 2012 & 2013 \\
\hline & Pobloción potencialmente protegible & \multirow{7}{*}{$\begin{array}{l}\text { FONDO GENERAL } \\
226.557 .936 € \\
80 \%\end{array}$} & $55,00 \%$ & $35,00 \%$ & $25,00 \%$ & $0,00 \%$ \\
\hline \multirow{12}{*}{$\begin{array}{l}\text { CRITERIOS } \\
\text { CORRECTORES }\end{array}$} & Dispersión geogrófice & & $0,60 \%$ & $0,60 \%$ & $0,60 \%$ & $0,60 \%$ \\
\hline & Insularidad & & $0,60 \%$ & $0,60 \%$ & $0,60 \%$ & $0,60 \%$ \\
\hline & Superficie & & $1,80 \%$ & $1,80 \%$ & $1,80 \%$ & $1,80 \%$ \\
\hline & $\begin{array}{l}\text { Beneficiarios reconocidos como } \\
\text { dependientes con derecho a prestación } \\
\text { y registrados en el SISAAD }\end{array}$ & & $613 \%$ & $63 \%$ & $63 \%$ & $63 \%$ \\
\hline & PIAs & & $189 \%$ & $38,90 \%$ & $48,90 \%$ & $73,90 \%$ \\
\hline & Prestaciones de Servicios & & $168 \%$ & $168 \%$ & $168 \%$ & $168 \%$ \\
\hline & $\begin{array}{c}\text { Renta per cápito disponible y pensión } \\
\text { media de jubilación }\end{array}$ & \multirow{3}{*}{$\begin{array}{c}\text { FONDO COMPENSACION } \\
28.319 .7 \angle 2 € \\
10 \%\end{array}$} & $49,00 \%$ & $49,00 \%$ & $49,00 \%$ & $49,00 \%$ \\
\hline & Coste de Servicios & & $49,00 \%$ & $49,00 \%$ & $49,00 \%$ & $49,00 \%$ \\
\hline & Emigrantes retomados & & $2,00 \%$ & $2,00 \%$ & $2,00 \%$ & $2,00 \%$ \\
\hline & $\begin{array}{l}\text { Formacion de Cuidadores No } \\
\text { Profesionales }\end{array}$ & \multirow{2}{*}{$\begin{array}{c}\text { FONDO CUMPLIMMENTO DE } \\
\text { ACUERDOS } \\
28.319 .742 € \\
10 \%\end{array}$} & $85 \%$ & $85 \%$ & $85 \%$ & $85 \%$ \\
\hline & $\begin{array}{c}\text { Transposicion de acuerdos a normativa } \\
\text { autonomica }\end{array}$ & & $15 \%$ & $15 \%$ & $15 \%$ & $15 \%$ \\
\hline & TOTAL & $283.197 .420 €$ & & & & \\
\hline
\end{tabular}

Fuente: Elaboración propia

Tabla 6: Comparación de índices de reparto del nivel acordado por CC.AA. (2007 a 2010)

COMPARACIÓN

\begin{tabular}{l} 
Andalucía \\
\hline Aragón \\
\hline Asturias (Principado de) \\
Balears (Illes) \\
Canarias \\
Cantabria \\
Castilla y León \\
Castilla-La Mancha \\
Cataluña \\
\hline Comunidad Valenciana \\
Extremadura \\
Galicia \\
Madrid (Comunidad de) \\
Murcia (Región de) \\
Navarra \\
\hline País Vasco \\
Rioja (La) \\
Ceuła y Melilla
\end{tabular}

\begin{tabular}{|c|c|c|c|}
\hline \multicolumn{4}{|c|}{ Índice de Distribución Resultante } \\
\hline 2007 & 2010 & 2010-2007 & Crec. \\
\hline 17.4918 & 21,6560 & 4,1642 & $23,81 \%$ \\
\hline 4,0442 & 3,8480 & $-0,1962$ & $-4,85 \%$ \\
\hline 3,4610 & 2,8826 & $-0,5784$ & $-16,71 \%$ \\
\hline 2,1260 & 1,9630 & $-0,1631$ & $-7,67 \%$ \\
\hline 3.7205 & 3,1035 & & \\
\hline 1,6333 & 1,7670 & 1337 & $8,19 \%$ \\
\hline 8,5900 & 7,9942 & $-0,5958$ & $-6,94 \%$ \\
\hline 5,6196 & 5,5857 & $-0,0340$ & $-0,60 \%$ \\
\hline 5.6324 & 17.4402 & 1,8078 & $11,56 \%$ \\
\hline 0.2682 & 8,93 & $-1,3364$ & $-13,01 \%$ \\
\hline 3,2038 & 2,9635 & $-0,2402$ & $-7,50 \%$ \\
\hline 9,0498 & 7,1405 & $-1,9093$ & $-21,10 \%$ \\
\hline 1.2942 & 10,3759 & $-0,9183$ & $-8,13 \%$ \\
\hline 2.8280 & 2,9888 & 0.1608 & $5,69^{\circ}$ \\
\hline & & & 42,54 \\
\hline & 0.2357 & -0.0133 & $-5,35$ \\
\hline
\end{tabular}

Fuente: Elaboración propia sobre información publicada en los BOE correspondientes a cada MCI 
c) La implementación en la Comunitat Valenciana (CV)

La singularidad en la aplicación de la ley en la CV se puede identificar con el confuso escenario institucional: múltiples agentes con competencias, con nuevas fórmulas de relación público-privadas, con una estructura institucional confusa y sometida a continuos cambios y regulaciones, y con el polémico papel en el procedimiento de reconocimiento del derecho de una empresa pública con forma jurídica mercantil, la Agencia Valenciana de Prestaciones Sociales S.A. (AVAPSA).

La información que se deriva del trabajo de campo etnográfico ha resultado coincidente con fuentes secundarias como los principales informes y estudios que realizaron un balance de la aplicación de la ley durante el marco temporal en el que se ha desarrollado dicho trabajo de campo ya fueran informes solicitados por las propias instancias gubernamentales, tales como el estudio realizado por la Comisión de Expertos (Cervera et al., 2009) nombrada por el pleno del Congreso de los Diputados a tal efecto y el informe que desde el Ministerio de Sanidad y Política Social se solicitó a la Agencia de Evaluación de Políticas Públicas (AEVAL, 2009) así como los promovidos desde organizaciones profesionales y afectados, siendo especialmente reveladores los Dictámenes que elabora la Asociación de Directores y Gerentes de SS.SS., los informes de los sindicatos por su condición de promotores de la ley e interlocutores en su desarrollo así como los informes que ha realizado el CERMI por su condición de representante de personas afectadas. Las memorias anuales de la Sindicatura de Greuges y del Defensor del Pueblo también se han considerado como valioso barómetro para medir la presión ciudadana sobre los incumplimientos así como la cantidad y cualidad de los mismos.

A diferentes niveles, y con diferentes enfoques, las fuentes secundarias analizadas y el testimonio de profesionales, ciudadanos/as y abogados, así como los datos primarios que ha publicado el SISAAD, han dado cuenta de la deficiente aplicación de la LAPAD en la CV remitiendo a factores como: un procedimiento largo y tortuoso con su expresión en la demora en la tramitación de los expedientes y en la percepción de las prestaciones; el no reconocimiento de la retroactividad; la paralización de expedientes por cualquier motivo (por no tener grado o nivel suficiente; por falta de documentación; por no haber elegido una prestación antes de hacer el PIA); cambios continuos en la regulación; baja proporción de solicitudes en función de la población potencialmente dependiente; régimen estricto de incompatibilidades; modelo de atención basado en el predominio de la prestación de cuidados en el entorno familiar y las residencias; desviaciones de la norma en lo relativo al silencio administrativo o los órganos responsables de la valoración; la falta de 
información sobre el procedimiento y el estado de tramitación; confusión y falta de una visión global del los/as profesionales de los servicios municipales de atención a la dependencia; en caso de errores en el procedimiento, pérdida de documentación o paralización de la tramitación, la ciudadanía ha tenido dificultades para identificar a los/as responsables dada la compleja maraña de instancias que participan, generándoles una importante indefensión

El problema de implementación de la ley en la Comunitat Valenciana no ha remitido sólo al conflicto competencial o a la estructura de financiación desplegada sino que se han añadido tres ingredientes más como claros obstáculos en el efectivo cumplimiento de la ley, los cuales vienen a verificar la hipótesis planteada puesto que son evidencias de una débil cultura de la legalidad: el modelo de política social desarrollado desde el que se articulaba la relación público-privado, la relación administración-ciudadanía (rendición de cuentas y transparencia) y la defensa del derecho en virtud a su judicialización. A continuación se profundiza en los mismos como expresión de la quiebra entre el plano formal del derecho y el real de la práctica política y administrativa.

\section{Débil cultura de la legalidad: razones de la disonancia entre el texto legal y la experiencia cotidiana en el ejercicio del derecho}

Una de las razones que ha tenido un impacto central en la contribución o menoscabo de la cultura de la legalidad ha sido la rendición de cuentas. Según Villoria y Wences (2010: 14), la rendición de cuentas podría definirse como un proceso a través del cual gobernantes, representantes y servidores públicos, informan, responden y justifican sus actos, sus decisiones y sus planes de acción a gobernados/as y se sujetan a las sanciones y recompensas. Durante este proceso, la ciudadanía observa, analiza, verifica y juzga su reconocimiento, critica o rechaza la actuación de la persona representante, gobernante o funcionaria pública haciendo uso de mecanismos e instrumentos de control institucional, electoral y/o social.

Así, la responsabilidad de rendir cuentas y solicitarla no se refiere únicamente a la acción de gobierno sino también a la acción administrativa. Algunos de los múltiples «fallos» de la administración valenciana en el procedimiento, que pueden calificarse como problemas de rendición de cuentas, han sido de carácter legal y administrativos.

Retomando las definiciones de estos conceptos, podremos afirmar que un gobierno y una administración que velen por una rendición de cuentas legal deben mostrar comportamientos acordes con los procedimientos legales y éstos contribuirán a una rendición de cuentas administrativa cuando los actos 
burocráticos (eficaces, eficientes, equitativos y transparentes) sean responsables y respetuosos con dichos procedimientos.

No tanto lo que la propia administración autonómica ha declarado, respondido, informado o justificado resultan ser evidencias de una débil o nula rendición de cuentas administrativa y legal como los datos revelados en el trabajo etnográfico referentes a las preocupaciones que tanto la ciudadanía como los/as profesionales, agentes sociales e incluso instituciones como el Defensor del Pueblo y el Síndic de Greuges han mostrado, generando preguntas, denuncias públicas o reivindicaciones, evidenciando además la ausencia de control ciudadano frente a los hechos relatados.

En definitiva, el trabajo etnográfico descubre que la ciudadanía se ha enfrentado a un complejo deambular, de «idas y venidas» entre diferentes organizaciones (especialmente AVAPSA) ${ }^{12}$ y administraciones, con largos plazos de espera y con exigua, confusa y cambiante información, tal y como muestra la siguiente síntesis:

A. Para valorar la rendición de cuentas administrativa se han identificado los siguientes fallos que se han mostrado como claros indicadores de su débil presencia:

a.1. Parte del proceso ha discurrido con irregularidades en la forma de proceder (por ejemplo, las notificaciones vía teléfono o email).

a.2. Se detectan irregularidades como la petición reiterada de la misma documentación, la pérdida de la misma y la paralización del procedimiento ante la falta de instrucciones claras.

a.3. Dilaciones en la resolución, paralización del procedimiento.

a.4. Silencio por respuesta: se ha impuesto el silencio administrativo negativo y, como consecuencia, la ciudadanía se ha mostrado insegura, desconcertada e indefensa.

a.5. Estructura fundamental de carácter local (Servicios Municipales de Atención a la Dependencia - SMAD) dependiente de una orden de ayudas sujeta a convocatorias de subvención anuales pudiéndose dar el caso de periodos en los que desaparece el servicio que debe dar respuesta a un derecho subjetivo hasta la renovación de la misma.

a.6. Múltiples agentes con competencias en el procedimiento (AVAPSA, SMADs y Conselleria) con directrices confusas, falta de coordinación y desconcierto de los/as profesionales.

12. AVAPSA constituye la empresa pública de gestión privada con forma jurídica de sociedad anónima que ha intervenido en alguna de las partes del procedimiento en el reconocimiento del derecho en la Comunidad Valenciana. 
a.7. Implantación limitada del catálogo de prestaciones y servicios. Protagonismo de la prestación del cuidador familiar y residencias.

B. Para valorar la rendición de cuentas legal se han encontrado los siguientes fallos que resultan ser indicadores de su débil presencia:

b.1. Generación de un sistema de provisión «híbrido» (público + privado) que ha intervenido en el espacio de gestión y que ha dado lugar al incumplimiento de normativa básica, aspecto éste que remitía al debate jurídico sobre si la empresa pública AVAPSA podía, o no, desempeñar funciones que suponen autoridad pública.

b.2. La participación de la mercantil AVAPSA ha implicado el no control sobre los procesos de contratación de personal para asegurar las condiciones básicas de «capacidad, mérito y publicidad».

b.3. Se ha incurrido en procesos de contratación que diluían, aún más, la responsabilidad pública.

b.4. Fuerte producción normativa a nivel central y autonómico que ha provocado cambios continuos en la estructura institucional, competencias, procedimientos, etc.

b.5. Desviaciones e irregularidades respecto a la norma: valoraciones de oficio y fuera del entorno habitual de la persona.

b.6. Disparidad territorial en la aplicación de los acuerdos que se han derivado del Consejo Territorial.

b.7. Indefensión jurídica: agotada la vía de la reclamación administrativa sin respuesta alguna, se ha de efectuar un recurso contencioso- administrativo, sometiéndose a un proceso aplazado en el tiempo y asumiendo costas judiciales que no todo el mundo puede afrontar.

b.8. Estricto régimen de incompatibilidades con otras prestaciones o servicios ya concedidos que incluso suponen un retroceso en derechos.

En definitiva, estos «fallos» en la relación administración - ciudadanía han servido para medir y operativizar la rendición de cuentas (legal y administrativa), revelando la debilidad de los siguientes componentes:

- La ausencia de transparencia sobre la información relevante: escasa divulgación de la ley, falta de información sobre el procedimiento, dificultad en el acceso a la información que consta en los expedientes y la no información pública sobre la función que ejerce AVAPSA en el sistema. La referencia a la escasa o nula información relativa al procedimiento y a los criterios que la administración ha empleado en el 
mismo ha sido redundante en el discurso de los juristas que trabajan en la defensa y garantía de este derecho en diferentes organizaciones.

- La ausencia de participación y nula capacidad de influir en las decisiones: débil responsabilidad pública que, a su vez, debilita el estatus de ciudadanía generando indefensión frente al silencio de la administración y escasa capacidad real de elección o desacuerdo con la prescripción profesional. También, en el mismo sentido y de manera contundente se han pronunciado todos/as los/as juristas entrevistados/as, haciendo énfasis en el silencio como respuesta habitual de la administración y los graves perjuicios que conlleva esta forma de proceder sobre el derecho (inseguridad, incertidumbre, menosprecio y falta de garantías al derecho).

La aplicación de la LAPAD en la Comunitat Valenciana ha resultado ser ejemplo de cómo los avances en el marco jurídico no necesariamente se materializan incluso cuando se refiere a derechos de obligado cumplimiento por parte de las administraciones.

Los colectivos de profesionales, la ciudadanía, las organizaciones de personas discapacitadas y mayores así como los agentes sociales han insistido en las ineficiencias propias de su puesta en marcha: los procedimientos empleados, las dificultades que se plantean en el ejercicio del derecho y las garantías de equidad según territorio de residencia.

La acción reivindicativa y propositiva de las plataformas ciudadanas, organizaciones de profesionales y sindicatos han dado muestras de una viva y rica rendición de cuentas societal pero sus posibilidades de injerencia en la acción gubernamental han sido mínimas, inclusive en el caso de aquellas organizaciones, como ocurre en el caso de los sindicatos, que forman parte de órganos como el Comité Consultivo. Las alegaciones a la norma valenciana, la recogida de firmas, las manifestaciones o concentraciones y los intentos de interlocución con la administración no han resultado efectivos al igual que tampoco lo han sido los organismos que velan por la rendición de cuentas horizontal.

Pese al escaso poder sancionador, sí que ha existido una contribución importante en términos de «información, respuestas y exigencia de responsabilidades» que no ha acabado sólo en el terreno judicial sino en el plano de la política y la acción democrática de la ciudadanía, siendo elementos imprescindibles para alimentar no sólo la cultura de la legalidad sino, fundamentalmente, la cultura democrática. En este sentido, se puede concluir que frente a una débil rendición de cuentas legal y administrativa se ha logrado una fuerte 
rendición de cuentas societal ${ }^{13}$ y horizontal ${ }^{14}$, con un importante papel de las plataformas ciudadanas, defensores del pueblo así como el propio tribunal de cuentas.

Por otro lado, la LAPAD, al reconocer un derecho subjetivo, ha motivado la defensa judicial de éste, siendo muchas personas quienes han emprendido su vía de reivindicación de manera individualizada y por la vía de los Tribunales. Si bien ésta es una respuesta legítima y necesaria, no se puede reducir la defensa del derecho a la misma y ni siquiera representa una vía interesante para alimentar la cultura de la legalidad y democrática.

La garantía de los derechos sociales no puede dirimirse únicamente en el terreno judicial porque, como plantea Pisarello (2007: 90), éste puede convertirse en un "factor de erosión de la propia participación política, puesto que generaría en los ciudadanos una «ilusión de tutela» que lo llevaría a remplazar la lucha electoral y la movilización social por la imposición de acciones judiciales». Por tanto, también deben ser responsables de su tutela órganos legislativos, ejecutivos incluso órganos independientes como los tribunales de cuentas o los defensores del pueblo. En conclusión, se trata, como plantea este autor, de una reconstrucción compleja de las garantías a través tanto de mecanismos institucionales como extrainstitucionales o sociales, facilitando la organización colectiva y la participación como mecanismos de autotutela.

Las referencias a los diferentes mecanismos de garantía y tutela de los derechos remiten inevitablemente a los diferentes niveles de rendición de cuentas reconocidos. Si éstos no se dan, se debilitan los mecanismos administrativos y políticos responsables de garantizar los derechos sociales.

Tras la investigación realizada, las respuestas apuntan a la escasa o nula cultura de la legalidad y al mal gobierno, manifestadas con claridad en la relación administración-ciudadanía. Se trata de buscar las razones estructurales que dan origen a estas situaciones y que, a modo de pescadilla que se muerde la cola, son a su vez causa y consecuencia: la desmaterialización del Estado de Bienestar estaría en la base de la frágil cultura de la legalidad con la que opera la administración pero, a su vez, ésta débil cultura contribuye a la desmaterialización del anterior. Como plantean Pisarello y Asens (2012: 142) «Si no se podía obligar a un gobierno a satisfacer todos los derechos sociales de la

13. La rendición de cuentas societal es un mecanismo no electoral, vertical, de control de las autoridades políticas que descansa en las acciones de un múltiple conjunto de asociaciones de ciudadanos y movimientos sociales.

14. La rendición de cuentas horizontal supone la relación entre el gobierno, la administración y los órganos independientes de la misma pero de carácter institucional, controlando su labor con auditorías, defensores, tribunales de cuentas, fiscalías, inspectores. 
noche al día, si cabía, en cambio, imponerle la obligación de no generar retrocesos arbitrarios en relación con las conquistas adquiridas».

\section{Bibliografía}

Agencia Estatal de Evaluación de las Políticas Públicas y la Calidad de los Servicios (2009). La participación de la Administración General del Estado en el Sistema para la Autonomía y Atención a situaciones de Dependencia. http://www. aeval.es/comun/pdf/evaluaciones/E13.pdf (12 de julio de 2013).

Asociación Estatal de Directores y Gerentes de Servicios Sociales (2009). II Dictamen del Observatorio. Análisis y evaluación del proceso de implantación de la Ley de promoción de autonomía personal y atención a situaciones de dependencia. Ed. Ayuntamiento de Alcorcón.

Asociación Estatal de Directores y Gerentes de Servicios Sociales (2010a). IV Dictamen del Observatorio. Análisis y evaluación del proceso de implantación de la Ley de promoción de autonomía personal y atención a situaciones de dependencia. Enero. Ed. Ayuntamiento de Alcázar de San Juan.

Asociación Estatal de Directores y Gerentes de Servicios Sociales (2010b). V Dictamen del Observatorio. Análisis y evaluación del proceso de implantación de la Ley de promoción de autonomía personal y atención a situaciones de dependencia. Junio. Ed. Ayuntamiento de Alcorcón.

Asociación Estatal de Directores y Gerentes de Servicios Sociales (2011). VI Dictamen del Observatorio. Análisis y evaluación del proceso de implantación de la Ley de promoción de autonomía personal y atención a situaciones de dependencia. Ed. Colexio Oficial de Traballo Social de Galicia.

Asociación Estatal de Directores y Gerentes de SS.SS. (2008). I Dictamen del Observatorio. Evaluación y análisis de los procesos de implantación de la Ley de Promoción de autonomía personal y atención a situaciones de dependencia. Síntesis de las Jornadas celebradas en Alcázar de San Juan. Ed. Diputación de Ciudad Real.

Asociación Estatal de Directores y Gerentes de SS.SS. (2009b). III Dictamen del Observatorio. Desarrollo e implantación de la Ley de promoción de la autonomía y atención a personas en situaciones de dependencia. Junio 2009. Síntesis de las jornadas celebradas el 22 y 23 de mayo de 2009.

Cervera, M., Herce, J. A., LóPez, G., Rodríguez, G. y Sosvilla, S. (2009). Informe final del grupo de expertos para la evaluación del desarrollo y efectiva aplicación de la ley 39/2006 de promoción de la autonomía personal y atención a las personas en situación de dependencia. Ministerio de Sanidad y Política Social. http://www.imserso.es/InterPresent1/groups/imserso/documents/binario/informesaadgexpertos.pdf (19 de julio de 2013). 
GINER, S. (2005). Ciudadanía pública y sociedad civil republicana. Documentación Social, $n^{\circ} 139,13-34$.

KAHALE, D. T. (2009). La cobertura de la situación de dependencia. Madrid: Fundación Alternativas.

PIÉ, A. (2012). Fundamentos de la educación para la autonomía: los Disability Studies. En Pié, A. (coord.): Deconstruyendo la dependencia. Propuesta para una vida independiente (pp. 21-46). Barcelona: Editorial UOC.

PIÉ, A. (coord.) (2012). Deconstruyendo la dependencia. Propuestas para una vida independiente. Barcelona: Editorial UOC.

Pisarello, G. (2007). Los derechos sociales y sus garantías. Madrid: Trotta.

PisArello, G. y Asens, J. (2012). No hay derecho(s). La ilegalidad del poder en tiempos de crisis. Barcelona: Icaria.

ROLDÁN, E. (2001). ¿Hacia un sistema mixto del bienestar social? La evolución de los servicios sociales en España. Madrid: Editorial Complutense.

ROMAÑACH, J (2012). Ética y derechos en la práctica diaria de la atención a la dependencia: autonomía moral vs autonomía física. En A. Pié (coord.). Deconstruyendo la dependencia. Propuesta para una vida independiente (pp. 47-74). Barcelona: Editorial UOC.

SENNETT, R. (2003). El respeto. Madrid: Anagrama.

Vidriales, R., de Araoz, I., Beotas, J. L., López, J. M., Marrero, R. y Rodríguez, A. (2009). Análisis de los desarrollos normativos del SAAD. Colección Cermi. es, $n^{\circ}$ 40. Madrid: Cinca. http://www.cermi.es/es-ES/ColeccionesCermi/Cermi.es/Lists/Coleccion/Attachments/65/AnalisisdelosdesarrollosnormativosdelSAAD.pdf (10 de febrero de 2010).

VILLORIA, M. y WenCES, M. I. (2010). Cultura de la legalidad. Madrid: Catarata.

VV.AA. (2011). Informe del Gobierno para la evaluación de la Ley de promoción de autonomía personal y atención a situaciones de dependencia. Ministerio de Sanidad y Política Social.

Young, I. M. (2000). Inclusion and Democracy. Oxford: Oxford University Press. 